\title{
The correlation between mobile phone addiction and coping style among Chinese adolescents: a meta-analysis
}

\author{
Guang-Li Lu ${ }^{1 \dagger}$, Yue-Ming Ding ${ }^{2 \dagger}$, Yi-Ming Zhang ${ }^{2}$, Hai-Tao Huang ${ }^{2}$, Yi-Pei Liang ${ }^{1}$ and Chao-Ran Chen ${ }^{2 *}$
}

\begin{abstract}
Background: Mobile phone addiction has become a social problem that affects the healthy growth of adolescents, and it may be correlated with coping style. The aim of this study was to investigate the relationship between mobile phone addiction and coping style and the influencing factors for adolescents.

Methods: A meta-analysis was conducted by searching China National Knowledge Infrastructure (CNKI), WANFANG DATA and Chongqing VIP Information Co., Ltd. (VIP), PubMed, Web of Science, Embase, and PsycINFO. Stata 16.0 was used to analyse the overall effect and test the moderating effect.

Results: Thirty-three studies were included, involving a total of 20,349 subjects. There was no significant correlation between adolescents' mobile phone addiction and positive coping style $(r=-0.02,95 \% \mathrm{Cl}=-0.06$ to $0.02, P>0.05)$, but there was a moderate positive correlation between adolescents' mobile phone addiction and negative coping style $(r=0.31,95 \% \mathrm{Cl}=0.26$ to $0.36, P<0.001)$. The moderating effect analysis showed that the effect of dissertations on mobile phone addiction and positive coping style among adolescents was significantly larger than that of journal articles. The Smartphone Addiction Scale for College Students (SAS-C) showed the largest effect on mobile phone addiction and positive coping style among adolescents. The time of publication significantly positively moderated the relationship between mobile phone addiction and negative coping style among adolescents. The Simplified Coping Style Questionnaire (SCSQ) showed the largest effect on adolescents' mobile phone addiction and negative coping style. However, the correlation between adolescents' mobile phone addiction and coping style was not affected by age or gender.
\end{abstract}

Conclusions: There was a close relationship between mobile phone addiction and coping style among adolescents. In the future, longitudinal research should be carried out to better investigate the dynamic changes in the relationship between mobile phone addiction and coping style.

Keywords: Mobile phone addiction, Coping style, Adolescent, Meta-analysis

*Correspondence: kfccr@126.com

${ }^{\dagger}$ Guang-Li Lu was the first author and Yue-Ming Ding was the co-first author

${ }^{2}$ Institute of Nursing and Health, School of Nursing and Health, Henan University, Jinming Avenue, Kaifeng 475004, Henan, China

Full list of author information is available at the end of the article

\section{Background}

In recent years, with the rapid development of information technology, mobile phones have been integrated as an indispensable part of people's daily lives, with extremely rich functions. Their utilization rate is also increasing. According to the 47th China statistical report on Internet development, by the end of December 2020, China had 986 million mobile phone users, accounting for $99.7 \%$ of the total number of Internet 
users in China [1]. Although mobile phones make daily life more convenient, frequent use can also lead to mobile phone addiction $[2,3]$. Adolescents are in a critical period of behaviour change [4], and their low level of self-control and strong curiosity about new things make it easy for mobile phone use to lead to problems $[5,6]$. At present, mobile phone addiction has become a social problem that affects the healthy growth of adolescents. Relevant studies have shown that the prevalence of mobile phone addiction among Chinese adolescents exceeds 25\% [7], which seriously endangers their physical health $[8,9]$, mental health $[10,11]$, academic performance $[9,12,13]$ and social relations [14]. Therefore, to reduce the level of adolescents' mobile phone addiction, researchers have actively explored the causes and mechanisms of this addiction, and found that coping style constitutes an important external factor affecting this type of addiction in adolescents [15-17].

However, many researchers have drawn inconsistent conclusions about the relationship between coping style and adolescents' mobile phone addiction. Most studies have shown that there is a significant positive correlation between negative coping style and mobile phone addiction among adolescents. For example, $\mathrm{He}$ et al. [17] found that adolescents with high levels of loneliness were more inclined to adopt negative coping style, which leads to more serious problems with mobile phone addiction. However, other researchers found a significant negative correlation between negative coping style and mobile phone addiction among adolescents. For example, Quan et al. [18] found that adolescents who adopt negative coping style usually adjust their emotions by staying alone rather than using mobile phones, thus having a low level of mobile phone addiction. Similarly, there are several views on the relationship between positive coping style and mobile phone addiction among adolescents. Some studies found a significant negative correlation between them. For example, Chen et al. [19] found that positive coping styles such as problem solving and help seeking can inhibit mobile phone addiction in adolescents. Some studies found a significant positive correlation between them. For example, Quan et al. [18] found that adolescents who adopt positive coping style make full use of mobile phones to help them gain inspiration. Some studies showed no significant correlation between them. For example, Li et al. [20] found that adolescents who adopt positive coping style have more self-discipline, and mobile phones are only a tool for them to contact others and solve problems. Therefore, the first aim of the current meta-analysis was to investigate the relation between mobile phone addiction and coping style among adolescents.
As a secondary goal, we explored the potential moderators of effect sizes. Age, gender, publication type, year of publication and measurement tools were considered as potential moderators. First, some studies showed that there are differences in the degree of Internet addiction between middle school students and college students [21, 22]. Vocational college students (2-year or 3-year college students) have a stronger ability to resist pressure than undergraduates (4-year or 5-year college students) and are more inclined to choose positive coping styles, such as seeking help and problem solving, to cope with pressure [23-25]. Second, Dong et al. [26] found that when adolescents have problems such as mobile phone addiction, females are more flexible in changing their ways to adapt to pressure, while males, because they are not good at language expression and emotional communication, tend to choose negative coping styles such as escape in emotional regulation [27]. Third, generally, studies with significant results are often more likely to be published, so published journal articles may exaggerate the true relationship between variables [28]. Fourth, the emergence of mobile phone addiction has the characteristics of the times. With the popularization of the mobile internet and the emergence of diversified functions of smartphone, mobile phones have become an indispensable tool for modern people. Therefore, the relationship between adolescents' mobile phone addiction and coping style may also change over time. Finally, as far as the measurement of mobile phone addiction is concerned, the contents of several kinds of measurement tools are not the same. For example, the Smartphone Addiction Scale for College Students (SAS-C) [29] is based on the existing research and combined with the research on the addiction to smartphone applications (apps); thus, adding the two factors of app use and app updates might better reflect the current level of adolescents' mobile phone dependence. Similarly, in terms of the measurement of coping style, the Coping Style Questionnaire (CSQ) [30] and the Simplified Coping Style Questionnaire (SCSQ) [31] are widely used. The former divides coping styles into six categories: problem solving, self-blame, help seeking, fantasy, retreat and rationalization. The latter divides coping styles into two dimensions: positive and negative coping styles. Therefore, different tools for measuring coping style might also affect the relationship between adolescents' mobile phone addiction and coping style.

In view of this, this study conducted a meta-analysis of the correlation between mobile phone addiction and coping style among adolescents, deeply investigated the correlation between them, and explored the moderators affecting the relationship so as to provide an objective basis and direction for the effective prevention and treatment of mobile phone addiction among adolescents. 


\section{Methods}

\section{Search strategy}

A comprehensive search of Chinese and English literature was conducted to collect published studies related to mobile phone addiction and coping style at home and abroad. Two authors independently retrieved relevant studies from the China National Knowledge Infrastructure (CNKI), WANFANG DATA and Chongqing VIP Information Co., Ltd. (VIP), PubMed, Web of Science, Embase, and PsycINFO. Search terms used for mobile phones included "cell phone", "mobile phone", "smart phone", "smartphone", and "cellular phone". Search terms used for addiction included "addiction", "dependence", "use", "abuse", "dependency", “addicted to", "overuse", "problem use", and "compensatory use". Search terms used for coping style included "coping style", "coping styles", "coping mode", "coping strategy", and "coping strategies". Other search terms included "problematic cell phone use", "problematic mobile phone use", "problematic smart phone use", "problematic smartphone use", and "problematic cellular use". In addition, this study traced the references included in the identified studies and supplements as well as Chinese and English key words to identify relevant literature. The retrieval time spanned from the creation of the database to March 18, 2021.

\section{Inclusion and exclusion criteria}

The inclusion criteria were as follows: (a) the study design was a cross-sectional survey; (b) a clear scale was used to assess mobile phone addiction and coping style; (c) the correlation coefficient between mobile phone addiction and positive coping style or negative coping style was reported, and if the correlation coefficient of the total score was not reported, the full factor correlation coefficient should be reported; (d) the subjects were healthy adolescents; and (e) both published articles and dissertations were included. The exclusion criteria were as follows: (a) studies not published in Chinese or English; (b) studies with the same data published repeatedly; (c) studies with apparent data mistakes; and (d) studies with samples containing individuals with physical diseases or mental disorders.

\section{Coding variables}

The included articles were coded as follows: author, year of publication, region, sample size, gender, participant categories, publication type, correlation coefficient, mobile phone addiction scale and coping style scale (Table 1). For the input of the correlation coefficient, the coding standards were as follows: (a) If the correlation coefficient between coping style and mobile phone addiction is not reported but the values of $F, T$ and $X^{2}$ are reported, they are transformed into the $r$ value by the corresponding formula $\left(r=\sqrt{\frac{t^{2}}{t^{2}+d f}}\right.$, $d f=\mathrm{n}_{1}+\mathrm{n}_{2}-2 ; r=\sqrt{\frac{F}{F+d f_{e}}} ; r=\sqrt{\frac{\chi^{2}}{\chi^{2}+N}}$ ) [32]. (b) The study effect size was encoded as an effect size according to the independent samples. If the study contained multiple independent samples, the article effect size was coded separately. The studies that examined the correlation between mobile phone addiction and positive coping style and those that examined the correlation between mobile phone addiction and negative coping style were coded. (c) If only the correlation coefficients of certain dimensions between mobile phone addiction and coping style were reported, the average of each dimension was taken before coding.

\section{Quality assessment}

The literature quality evaluation was completed by two researchers independently. Any doubts or disagreement were resolved by centralized discussion (at least three people) or by soliciting the opinions of thirdparty experts. The nine-item Joanna Briggs Institution Critical Appraisal Checklist for Studies Reporting Prevalence Data was used to assess literature quality in this study [33]. The score for each item is zero ("no", "unclear" or "not applicable") or one ("Yes"), and the highest score is nine. Higher scores reflected better methodological quality.

\section{Data processing and analyses}

Stata 16.0 software was used for meta-analysis, and the correlation coefficient $r$ was used as the effect size in this study. Specifically, the $r$ value was first converted to the corresponding Fisher's $Z$ value by using the Fisher transform, weighted based on the sample size with $95 \%$ confidence intervals: $Z=0.5^{*} \ln [(1+r) /(1-r)]$, where the variance of $Z$ is $\mathrm{V}_{Z}=1 / \mathrm{n}-3$ and the standard deviation of $Z$ is $\mathrm{SE}_{Z}=$ square root of $(1 / \mathrm{n}-3)$. The evaluation criteria were as follows: when the correlation coefficient effect size $r \leq 0.10$, it was a low correlation; when $0.10<r<0.40$, the correlation was moderate; and when $r \geq 0.40$, the correlation was high [34]. Publication bias was analysed by funnel plots and Egger's linear regression test, and heterogeneity was examined with Cochran's $Q$ and $I^{2}$ statistics. When the $Q$ value was significant $(P<0.05)$ and $I^{2} \geq 75 \%$, this indicated a high degree of heterogeneity in the study, and it was more reasonable to choose the random effects model; otherwise, the fixed effects model was chosen [35]. In addition, subgroup analysis and sensitivity analysis were necessary to investigate the sources of heterogeneity. 
Table 1 Characteristics of the 33 studies included in the analysis

\begin{tabular}{|c|c|c|c|c|c|c|c|c|}
\hline \multirow[t]{2}{*}{ Name (year) } & \multirow[t]{2}{*}{ Region } & \multirow[t]{2}{*}{ Sample size } & \multirow[t]{2}{*}{ Female\% } & \multirow[t]{2}{*}{ Subject type } & \multirow[t]{2}{*}{ Publication type } & \multirow[t]{2}{*}{$R(\mathrm{PCS}$ and $\mathrm{NCS})$} & \multicolumn{2}{|c|}{ Measurement tool } \\
\hline & & & & & & & $\begin{array}{l}\text { Mobile phone } \\
\text { addiction }\end{array}$ & Coping style \\
\hline Quan (2014) [18] & Western & 546 & 0.55 & 2 & Dissertation & 0.12 and -0.25 & MPATS & CSQ \\
\hline Xu (2014) [38] & Eastern & 293 & 0.54 & 1 & Journal & 0.01 and 0.21 & MPDI & CCSS-MSS \\
\hline Wu (2015) [39] & Central & 687 & 0.61 & 3 & Dissertation & -0.01 and 0.29 & Self-compiled & SCSQ \\
\hline Zeng (2015) [40] & Central & 282 & 0.60 & 3 & Dissertation & 0.04 and 0.34 & MPATS & CSQ \\
\hline Chen (2015) [19] & Central & 421 & 0.53 & 3 & Journal & 0.19 and -0.17 & MPAI & CSQ \\
\hline Zhang (2015) [41] & Central & 307 & 0.69 & 3 & Journal & 0.11 and 0.33 & MPATS & SCSQ \\
\hline Li (2016) [20] & $\mathrm{N}$ & 696 & 0.71 & 3 & Journal & -0.03 and 0.31 & MPAI & SCSQ \\
\hline Zhang (2016) [42] & N & 276 & 0.70 & 3 & Journal & -0.14 and 0.39 & MPATS & SCSQ \\
\hline $\mathrm{Zu}(2016)$ [16] & Central & 548 & 0.60 & 3 & Journal & -0.01 and 0.32 & MPATS & CSQ \\
\hline Yan (2016) [43] & Eastern & 404 & 0.41 & 2 & Journal & $\mathrm{N}$ and 0.20 & MPAI & CSQ \\
\hline Wu (2016) [44] & Central & 624 & 0.55 & 2 & Dissertation & 0.15 and 0.27 & MPAI & HCSS-MSS \\
\hline Chen (2017) [45] & Central & 432 & 0.52 & 3 & Dissertation & 0.09 and 0.36 & MPAI & SCSQ \\
\hline Gao (2017) [46] & Western & 488 & 0.21 & 2 & Dissertation & 0.11 and 0.36 & MPAI & SCSQ \\
\hline Wang (2017) [47] & Central & 490 & 0.72 & 3 & Dissertation & $\mathrm{N}$ and 0.35 & SAS-C & SCSQ \\
\hline Xia (2017) [48] & Central & 330 & 0.64 & 3 & Journal & -0.17 and 0.34 & MPATS & CSQ \\
\hline Xin (2017) [49] & Central & 630 & 0.44 & 1 & Journal & -0.13 and 0.27 & MPDS-MSS & CCSS-MSS \\
\hline Zeng (2018) [50] & Central & 395 & 0.59 & 3 & Dissertation & -0.06 and 0.31 & MPATS & SCSQ \\
\hline $\mathrm{He}(2018)$ [17] & Central & 490 & 0.72 & 3 & Journal & $\mathrm{N}$ and 0.35 & SAS-C & SCSQ \\
\hline Liu (2018) [51] & Eastern & 728 & 0.79 & 3 & Journal & -0.01 and 0.19 & MPAI & WSCSQ \\
\hline Xiong (2018) [52] & Central & 359 & 0.60 & 3 & Journal & $\mathrm{N}$ and 0.30 & MPATS & SCSQ \\
\hline Sun (2018) [53] & M & 1041 & 0.44 & 1 & Journal & -0.13 and 0.35 & SAS-C & CCSS-MSS \\
\hline Xu (2018) [54] & Western & 316 & 0.53 & 1 & Journal & 0.07 and $\mathrm{N}$ & MPAS & SCOPE \\
\hline Zeng (2019) [55] & Eastern & 2178 & 0.49 & 2 & Journal & -0.01 and 0.26 & MPATS & SCSQ \\
\hline Xu (2019) [56] & $\mathrm{N}$ & 558 & 0.60 & 3 & Journal & -0.02 and 0.36 & MPAI & SCSQ \\
\hline Zhang (2019) [57] & Central & 1774 & 0.86 & 3 & Journal & -0.01 and 0.20 & MPAI & SCSQ \\
\hline Hong (2019) [58] & Eastern & 522 & 0.59 & 3 & Journal & -0.08 and 0.31 & MPAI & CSQ \\
\hline Han (2020) [59] & $\mathrm{N}$ & 366 & 0.57 & 3 & Dissertation & 0.08 and 0.73 & MPAI & SCSQ \\
\hline He (2020) [60] & M & 911 & 0.55 & 2 & Journal & -0.15 and 0.51 & MPATS & SCSQ \\
\hline Yuan (2020) [61] & Central & 870 & 0.77 & 3 & Dissertation & $\mathrm{N}$ and 0.38 & TMD-C & SCSQ \\
\hline Zheng (2020) [62] & $\mathrm{N}$ & 418 & 0.64 & 3 & Journal & -0.24 and 0.23 & SAS-CA & SCSQ \\
\hline Liu (2020) [63] & Eastern & 1169 & 0.44 & 3 & Journal & 0.03 and 0.43 & MPAI & SCSQ \\
\hline $\mathrm{He}(2020)$ [15] & M & 604 & 0.56 & 1 and 3 & Journal & $\mathrm{N}$ and 0.28 & MPAI & SCSQ \\
\hline Qiu (2021) [64] & Central & 190 & 0.68 & 3 & Journal & 0.07 and 0.27 & MPAI & SCSQ \\
\hline
\end{tabular}

$N$ Not reported, $M$ Mixed region, 1 Middle school students, 2 Vocational college students, 3 Undergraduate, PCS Positive coping, NCS Negative coping style, MPATS Mobile Phone Addiction Tendency Scale, MPDI Mobile Phone Dependence Inventory, MPAI Mobile Phone Addiction Index, SAS-C Smartphone Addiction Scale for College Students, MPDS-MSS Mobile Phone Dependence Scale for Middle School Students, MPAS Smartphone addiction scale, TMD-C the Test of Mobile Phone Dependence for Chinese Adolescents, SAS-CA Smartphone Addiction Scale for Chinese Adults, CSQ Coping Style Questionnaire, SCSQ the Simplified Coping Style Questionnaire, CCSS-MSS Chen's Coping Style Scale for Middle School Students, HCSS-MSS Huang's Coping Style Scale for Middle School Students, WSCSQ Wang's Simplified Coping Style Questionnaire, SCOPE the Student Coping Instrument

\section{Results}

\section{Basic characteristics of the included studies and quality assessment}

A total of 884 articles were initially retrieved in this study. After removing duplicates, the search produced 820 studies. A total of 762 studies were excluded based on titles and abstracts. Then, the full texts of 58 studies were assessed for eligibility, and 33 studies were ultimately included (see Fig. 1). Those included studies were published between 2014 and 2021. Among them, there were 10 dissertations and 23 journal articles. Collectively, 20,349 participants were enrolled in those studies, including 8318 males and 12,031 females. The largest sample size was 2178 , and the smallest was 190 . All studies used self-report scales to measure both mobile phone addiction and coping style. The most frequently used 


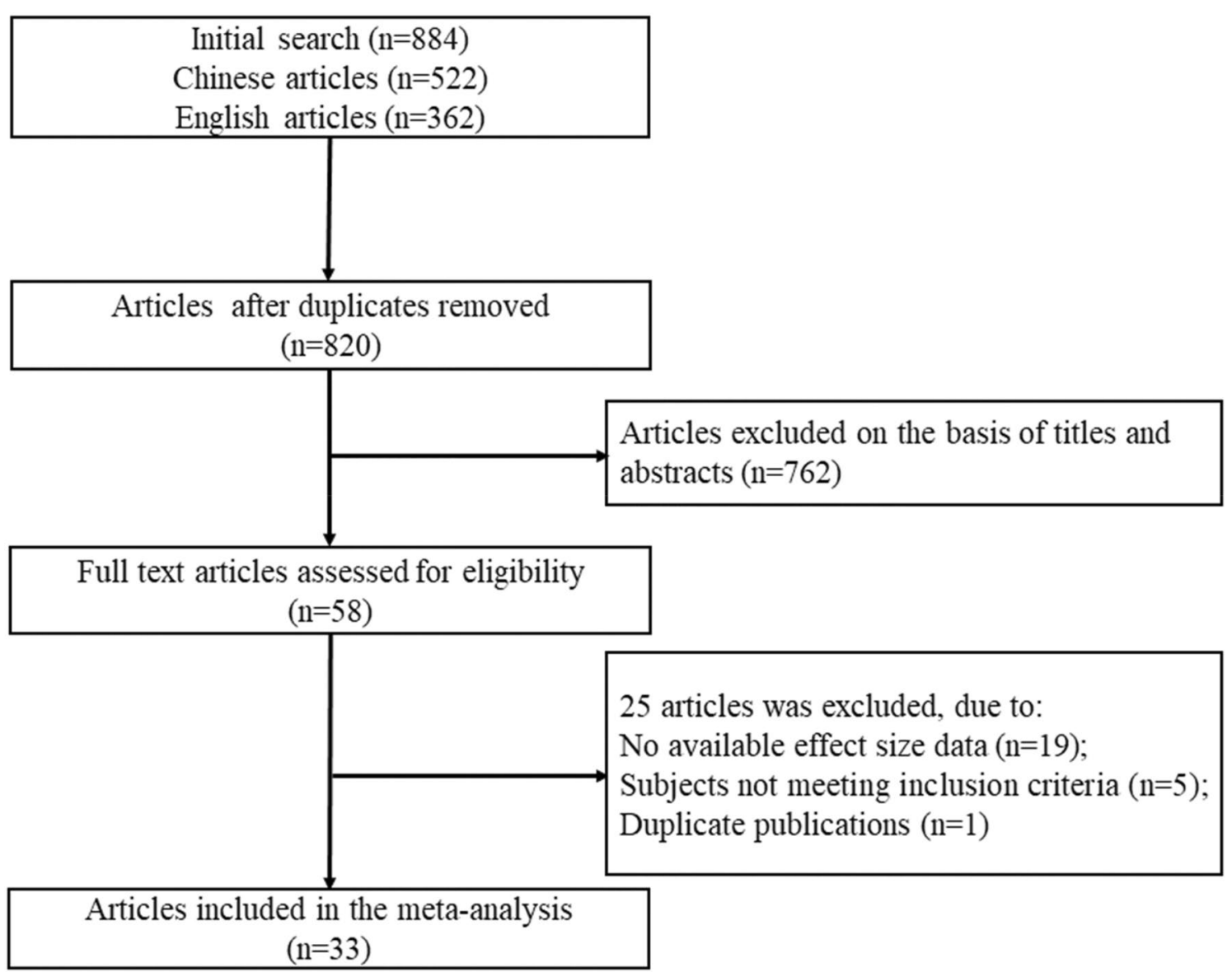

Fig. 1 The flow chart of the study selection process

measures of mobile phone addiction were the Mobile Phone Addiction Index (MPAI) [36] and Mobile Phone Addiction Tendency Scale (MPATS) [37]. Similarly, the most common instruments used for assessing coping style were the Simplified Coping Style Questionnaire (SCSQ) [31] and the Coping Style Questionnaire (CSQ) [30] (Table 1). In general, the quality of the included studies was at a medium or high level. The results from the quality assessment of each study can be found in Additional file 1: Table S1.

\section{Effect size and heterogeneity test}

A heterogeneity test was conducted on the included effect sizes, and the results showed that the $Q$ values of positive and negative coping styles were $140.27(P<0.001)$ and $474.33(P<0.001)$, respectively, and the $I^{2}$ values were $81.5 \%$ and $93.5 \%$, respectively, both higher than the $75 \%$ rule proposed by Higgins et al. [65], indicating a high level of heterogeneity among studies. Therefore, the random effects model was selected for meta-analysis. The results also suggest that it is necessary to rationally explore the moderating variables that affect the relationship between them.

The random effects model showed no significant correlation between positive coping style and mobile phone addiction among adolescents and a moderate positive correlation between negative coping style and mobile phone addiction among adolescents (positive coping style: $r=-0.02,95 \% \mathrm{CI}=-0.06$ to $0.02, P>0.05$; negative coping style: $r=0.31,95 \% \mathrm{CI}=0.26$ to $0.36, P<0.001$ ) (Table 2).

\section{Publication bias and sensitivity analysis}

First, the meta-analysis was tested by funnel plot for publication bias. Figures 2 and 3 show that the effect sizes of the relationship between mobile phone addiction and positive coping style and negative coping style of adolescents were basically evenly distributed on both sides of the overall effect sizes, indicating that the risk of publication bias was small in the study. Second, Egger's linear regression tests found that the $P$ values of positive coping style $(P=0.82)$ and negative coping style $(P=0.65)$ 
Table 2 Effect size and its heterogeneity test and publication bias test

\begin{tabular}{|c|c|c|c|c|c|c|c|c|c|c|}
\hline \multirow[t]{2}{*}{ Outcome Variable } & \multirow[t]{2}{*}{$k$} & \multirow[t]{2}{*}{$N$} & \multirow[t]{2}{*}{$r$} & \multicolumn{3}{|c|}{ Heterogeneity test } & \multicolumn{4}{|c|}{ Publication bias test } \\
\hline & & & & $Q$ & $d f$ & $I^{2}$ & $\begin{array}{l}\text { Egger's } \\
\text { intercept }\end{array}$ & $S E$ & $95 \% \mathrm{Cl}$ & $P$ \\
\hline Positive coping style & 27 & 17,132 & -0.02 & $140.27^{* *}$ & 26 & $81.5 \%$ & 0.36 & 1.52 & {$[-2.77,3.48]$} & 0.82 \\
\hline Negative coping style & 32 & 20,033 & $0.31^{* *}$ & $474.33^{* *}$ & 31 & $93.5 \%$ & 1.13 & 2.49 & {$[-3.96,6.22]$} & 0.65 \\
\hline
\end{tabular}

**P $<0.01$

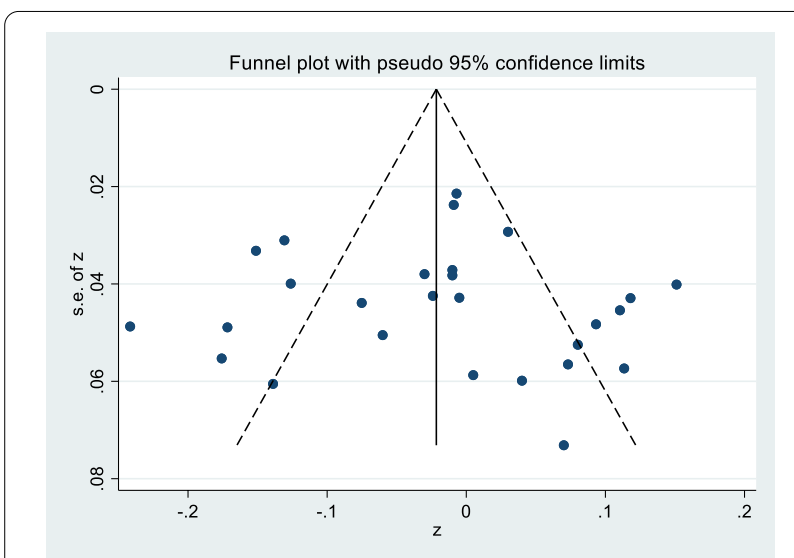

Fig. 2 Funnel plot of the correlation of mobile phone addiction and positive coping style

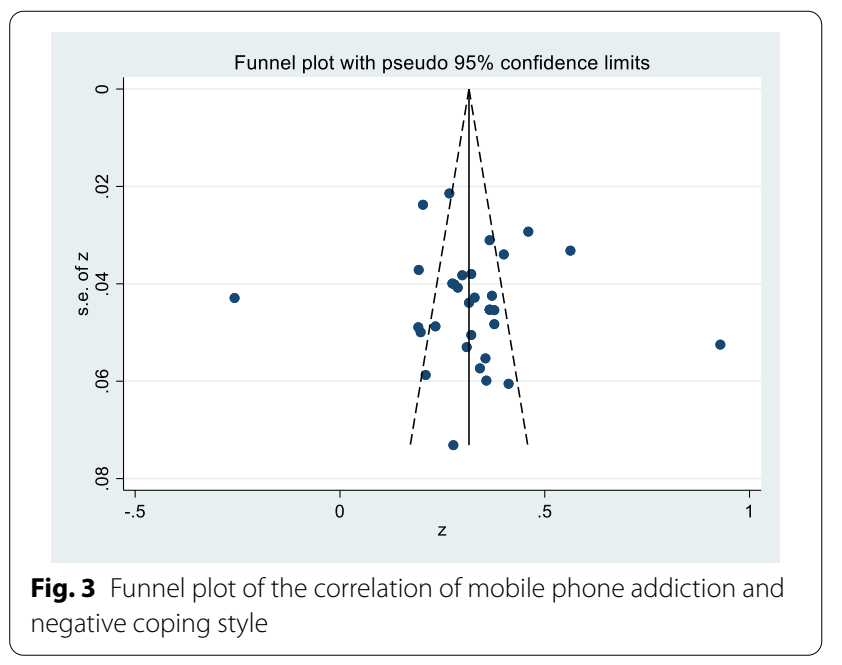

were greater than 0.05 , which further indicated that there was no publication bias in this study, and the estimated results of meta-analysis were relatively reliable. The sensitivity analysis of the included literature was conducted by culling one by one, and the results showed little change, indicating that the results of this study were relatively stable.

\section{Moderating effect test}

The heterogeneity of effects across studies was explored through moderator analysis. In this study, the moderating effect of 6 variables was tested: age, gender, publication type, year of publication, tool for measuring mobile phone addiction and tool for measuring coping style (Tables 3 and 4). The results showed that in terms of publication type, the effect of dissertations on mobile phone addiction and positive coping style among adolescents was significantly larger than that of journal articles (dissertations: $r=0.07,95 \% \mathrm{CI}=0.02$ to $0.12, P<0.05$; journal articles: $r=-0.05,95 \% \mathrm{CI}=-0.09$ to -0.02 , $P<0.001)$. In the tool for measuring mobile phone addiction, the SAS-C had the largest effect on mobile phone addiction and positive coping style among adolescents $(r=-0.13,95 \% \mathrm{CI}=-0.19$ to $-0.07, P<0.001)$. For the year of publication, the time of publication significantly positively moderated the relationship between mobile phone addiction and negative coping style $(P<0.05)$. In the tool for measuring coping style, the SCSQ had the largest effect on adolescents' mobile phone addiction and negative coping style $(r=0.37,95 \% \mathrm{CI}=0.31$ to $0.44, P<0.001)$. However, the moderating effects of age and gender on adolescents' mobile phone addiction and coping style were not significant (all $P>0.05$ ). Therefore, the meta-analyses presented a high level of heterogeneity between studies, mainly stemming from the publication type, year of publication, tool for measuring mobile phone addiction and tool for measuring coping style.

\section{Discussion}

The results showed that there was no significant correlation between mobile phone addiction and positive coping style $(r=-0.02, P>0.05)$, but there was a moderate positive correlation between mobile phone addiction and negative coping style $(r=0.31, P<0.001)$. This suggests that adolescents who are more addicted to mobile phones tend to adopt negative coping styles such as avoidance, fantasy, and denial to maintain their inner balance when facing pressure, which is consistent with previous studies. He et al. [17] and Li et al. [66] found that adolescents with high levels of loneliness were not very sociable in daily life and were prone to feeling helpless, which made 
Table 3 Coping style and mobile phone addiction: Univariate analysis of variance for moderator variables (categorical variables)

\begin{tabular}{|c|c|c|c|c|c|c|c|}
\hline Moderators & $\begin{array}{l}\text { Between-group } \\
\text { effect }\left(Q_{B E T}\right)\end{array}$ & $k$ & $N$ & $r$ & $95 \% \mathrm{Cl}$ & $\begin{array}{l}\text { Homogeneity test within } \\
\text { each group }\left(Q_{w}\right)\end{array}$ & $P^{2}$ \\
\hline \multicolumn{8}{|l|}{ Positive coping style } \\
\hline Participants type & 0.50 & & & & & & \\
\hline Middle school students & & 6 & 3635 & 0.01 & {$[-0.10,0.12]$} & $48.96^{* * *}$ & 89.8 \\
\hline Vocational college students & & 3 & 3392 & -0.02 & {$[-0.15,0.11]$} & $26.26^{* * *}$ & 92.4 \\
\hline Undergraduate & & 18 & 10,105 & -0.03 & {$[-0.07,0.01]$} & $64.73^{* * *}$ & 73.7 \\
\hline Proportion of women & 0.67 & & & & & & \\
\hline$\geq 0.6$ & & 12 & 6800 & -0.04 & {$[-0.08,0.01]$} & $40.24^{* * *}$ & 72.7 \\
\hline$<0.6$ & & 15 & 10,736 & -0.01 & {$[-0.06,0.05]$} & $99.16^{* * *}$ & 85.9 \\
\hline Publication type & $13.89^{* * *}$ & & & & & & \\
\hline Journal & & 19 & 13,716 & -0.05 & {$[-0.09,-0.02]$} & $82.73^{* * *}$ & 78.2 \\
\hline Dissertation & & 8 & 3820 & 0.07 & {$[0.02,0.12]$} & $17.66^{*}$ & 60.4 \\
\hline MPA measurement & $14.60^{* *}$ & & & & & & \\
\hline MPATS & & 9 & 5773 & -0.03 & {$[-0.10,0.04]$} & $44.87^{* * *}$ & 82.2 \\
\hline MPAI & & 12 & 8372 & 0.02 & {$[-0.03,0.06]$} & $43.37^{* * *}$ & 74.6 \\
\hline SAS-C & & 1 & 1041 & -0.13 & {$[-0.19,-0.07]$} & 0.00 & $\mathrm{~N} / \mathrm{A}$ \\
\hline Others & & 5 & 2350 & -0.06 & {$[-0.17,0.04]$} & $24.88^{* * *}$ & 83.9 \\
\hline CS measurement & 0.36 & & & & & & 0.28 \\
\hline CSQ & & 6 & 2649 & -0.04 & {$[-0.14,0.05]$} & $29.90^{* * *}$ & 83.3 \\
\hline SCSQ & & 15 & 10,851 & -0.01 & {$[-0.06,0.03]$} & $68.53^{* * *}$ & 79.6 \\
\hline Others & & 6 & 3632 & -0.01 & {$[-0.10,0.09]$} & $40.68^{* * *}$ & 87.7 \\
\hline \multicolumn{8}{|l|}{ Negative coping style } \\
\hline Participants type & 1.82 & & & & & & \\
\hline Middle school students & & 5 & 3076 & 0.31 & {$[0.25,0.36]$} & $9.55^{*}$ & 58.1 \\
\hline Vocational college students & & 4 & 4039 & 0.19 & {$[-0.10,0.49]$} & $230.15^{* * *}$ & 98.7 \\
\hline Undergraduate & & 22 & 12,314 & 0.35 & {$[0.29,0.41]$} & $215.06^{* * *}$ & 90.2 \\
\hline Proportion of women & 0.01 & & & & & & \\
\hline$\geq 0.6$ & & 16 & 9009 & 0.32 & {$[0.28,0.36]$} & $49.41^{* * *}$ & 69.6 \\
\hline$<0.6$ & & 16 & 11,024 & 0.32 & {$[0.22,0.42]$} & $422.08^{* * *}$ & 96.4 \\
\hline Publication type & 0.13 & & & & & & \\
\hline Journal & & 22 & 14,853 & 0.31 & {$[0.27,0.36]$} & $147.13^{* * *}$ & 85.7 \\
\hline Dissertation & & 10 & 5180 & 0.34 & {$[0.18,0.51]$} & $326.61^{* * *}$ & 97.2 \\
\hline MPA measurement & 3.69 & & & & & & \\
\hline MPATS & & 10 & 6132 & 0.30 & {$[0.16,0.44]$} & $240.01^{* * *}$ & 96.3 \\
\hline MPAI & & 14 & 8976 & 0.34 & {$[0.25,0.43]$} & $212.90^{* * *}$ & 93.9 \\
\hline SAS-C & & 3 & 2021 & 0.37 & {$[0.32,0.41]$} & 0.00 & N/A \\
\hline Others & & 5 & 2904 & 0.29 & {$[0.22,0.36]$} & $13.28^{*}$ & 69.9 \\
\hline CS measurement & $6.51^{*}$ & & & & & & \\
\hline CSQ & & 7 & 3053 & 0.21 & {$[0.04,0.39]$} & $144.03^{* * *}$ & 95.8 \\
\hline SCSQ & & 20 & 13,664 & 0.37 & {$[0.31,0.44]$} & $246.85^{* * *}$ & 92.3 \\
\hline Others & & 5 & 3316 & 0.27 & {$[0.20,0.34]$} & $14.62^{* *}$ & 72.6 \\
\hline
\end{tabular}

${ }^{*} P<0.05,{ }^{* *} P<0.01,{ }^{* * *} P<0.001$

Table 4 Univariate regression analysis of continuous variables (random effect model)

\begin{tabular}{llllll}
\hline Moderators & $\boldsymbol{k}$ & SE & $\boldsymbol{t}$ & $\mathbf{9 5 \%} \mathbf{C l}$ & $\boldsymbol{P}$ \\
\hline Positive coping style (year) & 27 & 0.01 & -1.08 & {$[-0.03,0.01]$} & 0.29 \\
Negative coping style (year) & 32 & 0.01 & 2.76 & {$[0.01,0.07]$} & 0.01 \\
\hline
\end{tabular}

them more inclined to adopt negative coping styles such as avoidance and fantasy. Wu et al. [44] found that adolescents with negative coping tend to feel psychologically uncomfortable in the face of pressure and dilemma, and the convenience and entertainment of mobile phones can be used as a way to vent about their bad experience 
so as to temporarily ignore the distress caused by their problems in life. Moreover, according to the theories of compensatory internet use and coping and defence mechanisms, the more pressure adolescents feel, the more likely they are to use mobile phones to cope with the discomfort caused by that pressure [67, 68].

According to the results of the subgroup analyses, age did not have a moderating effect on the relationship between adolescents' mobile phone addiction and coping style, perhaps because middle school students, vocational college students and undergraduates are all in a social environment where mobile networks and smartphones are widely available. Even for middle school students, approximately $80 \%$ own mobile phones, and nearly $40 \%$ of them use mobile phones without time limitations [69]. The external environment has the same influence on them. Therefore, there is little difference in coping styles when they encounter pressure or difficulties. Similarly, gender did not significantly moderate the relationship between adolescents' mobile phone addiction and coping style, which was inconsistent with the results of some previous studies. Some studies showed that compared with females, males were more likely to adopt negative coping style, to experience more pressure, and to be addicted to mobile phones $[15,60]$. Some studies also showed that females adopted more negative coping styles than males $[64,70,71]$. The reason for this difference might be that mobile phones meet different needs for males than for females.

Publication type significantly moderated the relationship between mobile phone addiction and coping style among adolescents. The correlation coefficients between adolescents' mobile phone addiction and positive coping style reported in different types of literature were different, and the degree of correlation reported in dissertations was higher than that reported in journal articles. This result is inconsistent with the claims that in meta-analysis studies with publication bias, the effect size of journal articles is larger than that of dissertations [72]. This difference might be related to the quality of the study and the rigor of the review and to the expectation of a difference in the study results.

Year of publication significantly moderated the negative correlation between adolescents' mobile phone addiction and negative coping style. The change trend of the correlation coefficient also indicated that the more recently the literature was published, the higher the correlation between mobile phone addiction and negative coping style among adolescents. This might be because with the popularity of mobile internet and smartphones, the multifunctional, interactive, entertainment and other characteristics of mobile phones not only cater to the psychological needs of adolescents, but also bring convenience and benefits to their daily life. Therefore, more and more adolescents spend most of their spare time on mobile phones [73], forming a habit of carrying or using mobile phones anytime and anywhere [74], which further increases the risk for mobile phone addiction, but also induce a series of anxiety, depression and other negative emotions [75]. When they face a difficult situation and lack sufficient support, they tend to adopt negative coping style to resist these negative emotions $[67,76]$. Adolescents who adopt negative coping style for a long time are prone to mobile phone dependence[15].

The tools for measuring mobile phone addiction significantly moderated the relationship between adolescents' mobile phone addiction and positive coping style but not the relationship between this addiction and negative coping style. The tools for measuring coping style significantly moderated the relationship between adolescents' mobile phone addiction and negative coping style, but the moderating effect on the relationship between this type of addiction and positive coping style was not significant. First, as far as the tools for measuring mobile phone addiction are concerned, the correlation between mobile phone addiction and positive coping style among adolescents reported by the SAS-C was the highest. A possible reason is that compared with other scales, the SAS-C was compiled for college students in 2014, based on the existing studies and compiled in conjunction with research on smartphone app addiction [29], so it better reflects the current level of adolescents' mobile phone dependence. Second, as far as the tools for measuring coping style are concerned, the effect of mobile phone addiction and negative coping style among adolescents reported by the SCSQ was the highest, which might be caused by the different dimensions subdivided by each scale. The SCSQ divided various coping styles into positive and negative dimensions according to their common characteristics, which were stable and balanced to a certain extent. However, the CSQ and the scales compiled by other scholars have problems such as the factor attribution of some items being unstable and unbalanced. Therefore, the SCSQ can better reflect the relationship between adolescents' mobile phone addiction and negative coping style than other scales can.

\section{Limitations and prospects}

Previous studies on the relationship between adolescents' mobile phone addiction and coping style have been inconsistent. In this study, the meta-analysis method was used to investigate the relationship between adolescents' mobile phone addiction and coping style, clarifying the controversy about the size and direction of the correlation between them in empirical studies. However, there are some limitations of this study. First, the studies 
included in the meta-analysis mainly focused on adolescents; research can be further expanded in the future to explore whether there are differences in the relationship between mobile phone addiction and coping style among different age groups. Second, this study only focused on the influence of some moderator variables on the relationship between mobile phone addiction and coping style, and other potential moderator variables should be analysed in the future, such as personality and cultural background. Finally, the studies included in this metaanalysis were cross-sectional, and longitudinal studies can be used in the future to establish the direction(s) of causality for the relationship between coping style and mobile phone addiction.

\section{Conclusion}

There was no significant correlation between adolescents' mobile phone addiction and positive coping style, while there was a moderate positive correlation between this type of addiction and negative coping style. Thus, adolescents with negative coping style had a higher degree of dependence on mobile phones. Publication type and mobile phone addiction measurement tools significantly moderated the relationship between adolescents' mobile phone addiction and positive coping style. Year of publication and coping style measurement tools significantly moderated the relationship between adolescents' mobile phone addiction and negative coping style, but the correlation between their mobile phone addiction and coping style was not moderated by age or gender. In the future, longitudinal studies should be carried out to better investigate the dynamic changes in the relationship between adolescents' mobile phone addiction and coping style.

\begin{abstract}
Abbreviations
MPATS: Mobile Phone Addiction Tendency Scale; MPDI: Mobile Phone Dependence Inventory; MPAl: Mobile Phone Addiction Index; SAS-C: Smartphone Addiction Scale for College Students; MPDS-MSS: Mobile Phone Dependence Scale for Middle School Students; MPAS: Smartphone addiction scale; TMD-C: The Test of Mobile Phone Dependence for Chinese Adolescents; SAS-CA: Smartphone Addiction Scale for Chinese Adults; CSQ: Coping Style Questionnaire; SCSQ: The Simplified Coping Style Questionnaire; CCSS-MSS: Chen's Coping Style Scale for Middle School Students; HCSS-MSS: Huang's Coping Style Scale for Middle School Students; WSCSQ: Wang's Simplified Coping Style Questionnaire; SCOPE: The Student Coping Instrument.
\end{abstract}

\section{Supplementary Information}

The online version contains supplementary material available at https://doi. org/10.1186/s13034-021-00413-2.

Additional file 1: Table S1. Quality assessment for the 33 studies in the current meta-analysis.
Acknowledgements

Not applicable.

\section{Authors' contributions}

GLL and YMD conceived and designed the study, YMD and HTH analyzed the data, GLL and YMD completed the original draft, GLL, YMZ, HTH and YPL revised the article, CRC and GLL reviewed, revised and edited the writing. All authors contribute sufficiently to this work. All authors read and approved the final manuscript.

\section{Funding}

This research was funded by the Graduate Education Innovation and Quality Improvement Program of Henan University, Grant Number SYL19060141; the Henan Provincial Social Science Planning Decision Consulting Project, grant number 2018JC38; and the Graduate Education Reform and Quality Improvement Project of Henan Province, grant number YJS2021AL074.

\section{Availability of data and materials}

The datasets used and/or analysed during the current study are available from the corresponding author on reasonable request.

\section{Declarations}

Ethics approval and consent to participate

Not applicable.

Consent for publication

Not applicable.

\section{Competing interests}

The authors declare that they have no competing interests.

\section{Author details}

'Institute of Business Administration, School of Business, Henan University, Kaifeng, Henan, China. Institute of Nursing and Health, School of Nursing and Health, Henan University, Jinming Avenue, Kaifeng 475004, Henan, China.

Received: 17 June 2021 Accepted: 11 October 2021

Published online: 15 October 2021

\section{References}

1. China Internet Network Information Center. http://www.cnnic.net cn/hlwfzyj/hlwxzbg/hlwtjbg/202102/P020210203334633480104.pdf. Accessed 3 Feb 2021.

2. Mahapatra S. Smartphone addiction and associated consequences: Role of loneliness and self-regulation. Behav Inform Technol. 2019;38(8):833-44.

3. Shen X, Wang JL. Loneliness and excessive smartphone use among Chinese college students: Moderated mediation effect of perceived stressed and motivation. Comput Hum Behav. 2019;95:31-6.

4. Mak KK, Lai CM, Watanabe H, Kim DI, Bahar N, Ramos M, et al. Epidemiology of internet behaviors and addiction among adolescents in six Asian countries. Cyberpsychol Behav Soc Netw. 2014;17(11):720-8.

5. Munno D, Cappellin F, Saroldi M, Bechon E, Guglielmucci F, Passera R, et al. Internet Addiction Disorder: Personality characteristics and risk of pathological overuse in adolescents. Psychiatry Res. 2017;248(9):1-5.

6. Wang J-L, Rost DH, Qiao R-J, Monk R. Academic stress and smartphone dependence among Chinese adolescents: a moderated mediation model. Child Youth Serv Rev. 2020;118:105029.

7. Tang J, Hao J, Han H, Wan Y, Li H, Gu X, et al. Relationship between mobile phone dependence and injury among Bengbu middle school students. Chin J Sch Health. 2016;37(2):208-11.

8. Alosaimi FD, Alyahya H, Alshahwan H, Al Mahyijari N, Shaik SA. Smartphone addiction among university students in Riyadh. Saudi Arabia Saudi Med J. 2016;37(6):675-83.

9. Mahapatra S. Smartphone addiction and associated consequences: role of loneliness and self-regulation. Behav Inform Technol. 2019;1:12. 
10. Kim SG, Park J, Kim HT, Pan Z, Lee Y, Mclntyre RS. The relationship between smartphone addiction and symptoms of depression, anxiety, and attention-deficit/hyperactivity in South Korean adolescents. Ann Gen Psychiatry. 2019;18:1.

11. Yang X, Zhou Z, Liu Q, Fan C. Mobile phone addiction and adolescents' anxiety and depression: The moderating role of mindfulness. J Child Fam Stud. 2019;28(3):822-30

12. Rozgonjuk D, Saal K, Täht K. Problematic smartphone use, deep and surface approaches to learning, and social media use in lectures. Int J Environ Res Public Health. 2018;15:1.

13. Samaha M, Hawi NS. Relationships among smartphone addiction, stress, academic performance, and satisfaction with life. Comput Hum Behav. 2016:57:321-5.

14. Jun S. The reciprocal longitudinal relationships between mobile phone addiction and depressive symptoms among Korean adolescents. Comput Hum Behav. 2016;58(May):179-86.

15. He A, Wan J, Ma R, Hui Q. Relationship between mobile phone addiction and negative coping style among adolescents: A cross-lagged study. Chinese J Clin Psychol. 2020;28(3):628-32.

16. Zu J, Zhang X, Zuo E, Ding X. College students'self-esteem and mobile phone dependence: The multiple mediating effects of coping styles. Chinese J Spec Educ. 2016;10:85-90.

17. He A, Wang C, Hui Q. Loneliness and smartphone dependence: The role of negative coping. Chinese J Clin Psychol. 2018;26(6):1222-5.

18. Quan K. Research on the cellphone addiction of advanced vocational college freshmen: Current situation and psychological causes (Master's thesis): China Master's Theses Full-text Database; 2014.

19. Chen X. A study on the current situation and influencing factors of mobile phone addiction among college students. J Kaifeng Vocat Coll Cult Art. 2015;35(4):169-71.

20. Li J, Yan G, Zhang J. Mobile phone dependence and alone feeling in medical college students: Coping style's mediating effect. China J Health Psychol. 2016;24(12):1828-31.

21. Zhang $X$, Luo Y. Internet addiction and the associated factors in adolescent in Yancheng. Chin J School Health. 2015;36(2):223-5.

22. Feng $R$. The study of group counseling intervention on student's network addiction by using core self-evaluation (Master's thesis): China Master's Theses Full-text Database; 2011.

23. Tang M. Difference of coping styles of psychological crisis stress among undergraduates and vocational college students. J Campus Life Ment Health. 2012;10(2):80-1.

24. Wang F, Gao S, Ye H, Zhou Z, Gao Y. Research on the features of college students' coping style and the relationship between college students' coping style and suicide ideation. Med Res Educ. 2013;30(3):59-65.

25. Wei L, Lei G. A comparative study on employment pressure sources and coping styles of undergraduates and vocational (junior college) students. J Jianghan U (Soc Sci Ed). 2012;29(2):95-9.

26. Dong Z, Zhang D. The relationship between psychological suzhi, emotion regulation strategies and life satisfaction among middle school students. J Southwest U (Soc Sci Ed). 2015;41(6):99-103.

27. Gullone E, Hughes EK, King NJ, Tonge B. The normative development of emotion regulation strategy use in children and adolescents: a 2-year follow-up study. J Child Psychol Psyc. 2010;51(5):567-74.

28. Sterne JA, Gavaghan D, Egger M. Publication and related bias in metaanalysis: power of statistical tests and prevalence in the literature. J Clin Epidemiol. 2000;53(11):1119-29.

29. Su S, Pan T, Liu Q, Chen X, Wang Y, Li M. Development of the smartphone addiction scale for college students. Chin Ment Health J. 2014;28(5):392-7.

30. Xiao J, Xu X. Study on validity and reliability of coping style questionnaire. Chin Ment Health J. 1996;4:164-8.

31. Xie Y. A preliminary study on the reliability and validity of the simple coping style scale. Chin J Clin Psychol. 1998;6(2):53-4.

32. Card NA. Applied Meta-Analysis for Social Science Research: Applied Meta-Analysis for Social Science Research; 2012.

33. Munn Z, Moola S, Lisy K, Riitano D, Tufanaru C. Methodological guidance for systematic reviews of observational epidemiological studies reporting prevalence and cumulative incidence data. Int J Evid-Based Health. 2015;13(3):147-53.
34. Lipsey MW, Wilson DB. The way in which intervention studies have "personality" and why it is important to meta-analysis. Eval Health Prof. 2001;24(3):236-54.

35. Huedo-Medina TB, Sánchez-Meca J, Marín-Martínez F, Botella J. Assessing heterogeneity in meta-analysis: Q statistic or 12 index? Psychol Methods. 2006;11(2):193-206.

36. Leung L. Linking psychological attributes to addiction and improper use of the mobile phone among adolescents in Hong Kong. J Child Media. 2008:2(2):93-113.

37. Xiong J, Zhou Z, Chen W, You Z, Zhai Z. Development of the mobile phone addiction tendency scale for college students. Chin Ment Health J. 2012;26(3):222-5.

38. Xu H, Bi X. Middle school students' mobile phone dependence and its related factors. Psychol Res. 2014;7(4):80-5.

39. Wu H. Research on the situation of undergraduate mobile phone addiction and its relationship with personality and coping style (Master's thesis): China Master's Theses Full-text Database; 2015.

40. Zeng Y. The relationship among college student adult attachment, coping style and mobile phone addiction tendency (Master's thesis): China Master's Theses Full-text Database; 2015.

41. Zhang J, Liu J, Li Y, Yang G. Study on the relationship between mobile phone addiction, social support, and personality characteristics among medical students. J Henan Med Coll. 2015;27(6):761-4.

42. Zhang J, Liu J, Li Y. A path analysis on the relationships among coping style, personality characteristics and mobile phone addiction tendency among college students. Prev Med. 2016;28(1):17-9.

43. Yan J, Cheng J. Relationship between vocational college student's mobile mhone addiction and its countermeasures. J Shenzhen Inst Inf Technol. 2016;14(4):89-92.

44. Wu X. Research on relationships of the vocational students personality traits, coping styles and mobile phone addiction (Master's thesis). China Master's Theses Full-text Database; 2016.

45. Chen $\mathrm{K}$. The research of the relationship among life meaning and coping style, mobile phone dependency in university student (Master's thesis). China Master's Theses Full-text Database; 2017

46. Gao Y. The relationship between cell phone dependence and loneliness, coping style of secondary vocational school student: A case study Yuxi industry, finance and trade school (Master's thesis). China Master's Theses Full-text Database; 2017.

47. Wang C. The relationship and intervention research of emotional adjustment, negative coping style and smartphone dependence in college student (Master's thesis): China Master's Theses Full-text Database; 2017.

48. Xia Y, He A. The relationship between mobile phone addiction and loneliness: The mediating role of coping style. J Neijiang Norm U. 2017;32(12):12-7.

49. Xin Z, Li Z, Wang L. Psychological analysis and regulation of mobile phone dependence among middle school students: Regression analysis based on survey data and related studies. J Chaohu U. 2017;19(2):160-4.

50. Zeng Y. The relationship between college students' personality traits and mobile phone dependence: The mediating role of time management inclinations and the moderating effect of coping styles (Master's thesis): China Master's Theses Full-text Database; 2018.

51. Liu G, Fan Y, Zhu D. On the relationship between perfectionism and college students' mobile phone addiction: the mediating role of coping style. J Qilu Norm U. 2018;33(6):29-35.

52. Xiong S, Yuan M, Zhang B, Li Y. Relationship between loneliness and mobile phone addiction: The mediating effect of negative emotion and negative coping. China J Health Psych. 2018;26(12):1857-61.

53. Sun J, Liu Q, Yu S. Child neglect, psychological abuse and smartphone addiction among Chinese adolescents: The roles of emotional intelligence and coping style. Comput Hum Behav. 2019;90:74-83.

54. Xu T, Wang H, Fonseca W, Zimmerman M, Rost D, Gaskin J, et al. The relationship between academic stress and adolescents' problematic smartphone usage. Addict Res Theory. 2019;27(2):162-9.

55. Zeng $F$, Jiang $M$. The relationship between mobile phone addiction and life events and coping styles of vocational college students. Econ Outlook Bohai Sea. 2019;6:146-7.

56. Xu W, Ye C, Fang Y. Relationship between sensation seeking and mobile phone addiction: Mediating role of coping styles. Psychol Res. 2019;12(3):245-51. 
57. Zhang S, Wang D, He Y, Liu D, Wang H. Mediating effect of negative coping style between stress and mobile phone dependence in nursing undergraduates. J Nurs. 2019;26(10):44-8.

58. Hong B, Wang R. Study on the relationship between mobile phone dependence, procrastination behavior and coping style of college students. J Tangshan Norm U. 2019;41(2):154-6.

59. Han R. The influence of academic anxiety and academic boredom on mobile phone addiction: The mediating role of coping style (Master's thesis): China Master's Theses Full-text Database; 2020.

60. He J, Liu X, Guo L, Li T, Fu M, Liu D. Effect of function of mobile phone use on the self-perceived stress and mobile phone addiction of high vocational students. Chinese J School Health. 2020:41(2):213-6.

61. Yuan M. Influence of Taiyin personality, alexithymia and coping style on mobile phone addiction of college students (Master's thesis): China Master's Theses Full-text Database; 2020.

62. Zheng $\mathrm{H}, \mathrm{Ma} J$. Correlationship of mobile phone addiction with coping style and physical exercise in college students. J Taishan Med Coll. 2020:41(9):695-8.

63. Liu F, Zhang Z, Chen L. Mediating effect of neuroticism and negative coping style in relation to childhood psychological maltreatment and smartphone addiction among college students in China. Child Abuse Neglect. 2020;106:104531.

64. Qiu Z, Mao H, Wu W, Yuan M, Zhang B, Shi Z. Influence of self-identity on mobile phone addiction: Mediating effect of negative coping style. J Bioeduc. 2021;9(1):6-10.

65. Higgins JP, Thompson SG, Deeks JJ, Altman DG. Measuring inconsistency in meta-analyses. Brit Med J. 2003;327(7414):557-60.

66. Li X, Tang H, Ming Q, Zhang H. Relationship between loneliness and self-harmony: Mediating role of coping styles. Chinese J Clin Psychol. 2014;22(3):530-2.

67. Diehl M, Chui H, Hay EL, Lumley MA, Grühn D, Labouvie-Vief G. Change in coping and defense mechanisms across adulthood: longitudinal findings in a European American sample. Dev Psychol. 2014;50(2):634-48.
68. Kardefelt-Winther D. A conceptual and methodological critique of internet addiction research: Towards a model of compensatory internet use. Comput Hum Behav. 2014;31(31):351-4.

69. Mai Q, Cao R. Investigation on the use of mobilephones in middle school students in Tianjin. J Tianjin Acad Educ Sci. 2019;4:83-8.

70. Hampel P, Petermann F. Age and gender effects on coping in children and adolescents. J Youth Adolescence. 2005;34(2):73-83.

71. Zhang Y, Lu G, Liu Y, Zhou Y. Mediating effect of self-identity on relationship between interpersonal adaptation and mobile phone addiction tendency in college students. Chin Ment Health J. 2017;31(7):568-72.

72. Pan $\mathrm{H}$, Zhang $M$, Huang J. Meta-analysis of relationship between mobile phone dependence and loneliness of college students. Occup Health. 2020;36(9):1272-6.

73. Han S, Kim KJ, Kim JH. Understanding nomophobia: Structural equation modeling and semantic network analysis of smartphone separation anxiety. Cyberpsychol Behav Soc Netw. 2017;20(7):419-27.

74. Kwon M, Lee JY, Won WY, Park JW, Min JA, Hahn C, et al. Development and validation of a smartphone addiction scale (SAS). PLOS ONE. 2013;8(2):e56936

75. Hu Y, Huang $H$, Zhang $Y$, Zhou $C$. The mediating effect of negative emotions between mobile phone dependence and cognitive failure. Chinese J Clin Psychol. 2017;25(6):1088-92.

76. Spielman R. The ego and the mechanisms of defence. Aust Nz J Psychiat. 2002;36(3):430-4

\section{Publisher's Note}

Springer Nature remains neutral with regard to jurisdictional claims in published maps and institutional affiliations.
Ready to submit your research? Choose BMC and benefit from:

- fast, convenient online submission

- thorough peer review by experienced researchers in your field

- rapid publication on acceptance

- support for research data, including large and complex data types

- gold Open Access which fosters wider collaboration and increased citations

- maximum visibility for your research: over $100 \mathrm{M}$ website views per year

At $\mathrm{BMC}$, research is always in progress.

Learn more biomedcentral.com/submissions 